\title{
04/29/02
}

\section{The Evolution of SINEs and LINEs in the genus Chironomus (Diptera)}

Ekaterina Papusheva ${ }^{1 *}$, Mary C. Gruhl ${ }^{2}$, Eugene Berezikov ${ }^{1 \dagger}$, Tatiana Groudieva ${ }^{1 \dagger}$, Svetlana V. Scherbik $^{1}$, Jon Martin ${ }^{3}$ and Alexander Blinov ${ }^{1}$, Gerald Bergtrom ${ }^{2}$

${ }^{1}$ Institute of Cytology and Genetics, Novosibirsk, Russia

${ }^{2}$ Dept. of Biological Sciences, University of Wisconsin-Milwaukee, Milwaukee, Wisconsin

${ }^{3}$ Genetics Department, University of Melbourne, Melbourne, Australia.

*Present address: Physiologisches Institut, Universitat Goettingen, Goettingen, Germany.

${ }^{\dagger}$ Present address: Technische Universität Hamburg-Harburg, Hamburg, Germany.

Address for correspondence and reprints: Gerald Bergtrom, Department of Biological

Sciences, University of Wisconsin-Milwaukee, P.O. Box 413, Milwaukee, WI 53201, USA. Email: bergtrom@csd.uwm.edu

Key words: non-LTR retrotransposon, LINE, SINE, Chironomus, co-evolution

Running Head: Evolution of chironomid LINEs and SINEs

Abbreviations: non-LTR, non-long-terminal-repeat; NJ, neighbor-joining; nt, nucleotides;

SINE, short interspersed nuclear element; LINE, long interspersed nuclear element. 


\begin{abstract}
PCR amplification of genomic DNA from 51 species of the family Chironomidae shows that most contain relatives of retrotransposons originally found in Chironomus thummi (LINE
\end{abstract} NLRCth1; SINE CTRT1). More than 300 cloned PCR products were sequenced. The amplified regions of LINEs (ORF2 of the reverse transcriptase gene) are highly similar, suggesting strong conservation of a functional gene. Amplified SINEs (399 nucleotides) are less conserved, consistent with an absence of selection on non-coding DNA, though SINEs were sufficiently similar to suggest recent transposition. LINE phylogenies support mitochondrial and nuclear gene phylogenies, indicating vertical descent of the LINEs. In contrast, the SINE tree topology is significantly different, indicating that SINE evolution does not track species evolution. In addition, many SINEs are cladistically misplaced on the tree. An analysis of diagnostic residues, based on species-specific nucleotide differences from a consensus sequence of aligned SINEs, reveals at least 8 different SINE subfamilies. Some species contain SINEs belonging to more than one subfamily, and SINEs of the same subfamily are found in different species. Many of the latter may 1) correspond to cladistically misplaced elements and may have been horizontally transferred (likely between more distantly related species), 2) may be artifacts of convergent evolution of a small number of informative nucleotides, or 3) may be descendants of ancient elements retained in some but lost in other species. Similar 3' sequence motifs of CTRT1 and NLRCth1 suggest that they are a co-evolving retrotransposon pair in which CTRT1 mobility depends on enzymes expressed during the transposition of NLRCth1. Phylogenetic separation of a LINEs and SINEs in the 'plumosus' species group further support that the elements of this clade are a co-evolving LINE/SINE pair distinct from elements in the rest of the species. 


\section{Introduction}

Reverse transcription, once thought peculiar to retrovirus replication, can also account for the loss of introns from split genes, the creation of processed pseudogenes and the transposition of SINEs and LINEs (Short and Long Interspersed Nuclear Elements, respectively). Ranging from 3000-7000 nucleotide (nt) pairs in length, LINEs (also called non-LTR retrotransposons or retrotransposons) are one of three kinds of transposable elements that encode reverse transcriptase as well as activities required for mobility (Xiong and Eickbush 1990). The others are the retroviruses themselves and Long Terminal Repeat (LTR) retrotransposons. At 80-400 nt, SINEs are derived from RNA polymerase III-catalyzed transcripts, including tRNAs in most organisms and 7SL RNA transcripts in primates and rodents (Alu and Bl elements, respectively). Unlike LINEs, SINEs do not encode polypeptides, and once transposed, most SINE copies are thought to be incapable of further transposition (Takasaki et al. 1994). Active SINEs and LINEs can result in the integration of hundreds or even thousands of retrotransposon copies, profoundly influencing the genomic landscape in animals and plants beyond the impact of a few viruses (reviewed in Britten 1997; Kazazian and Moran 1998; Kidwell and Lish 2000; Bennetzen 2000).

As of this writing, 15 LINE families are known (Malik et al. 1999; Malik and Eickbush 1999; Volff et al. 2000; Lovesin et al. 2001; Archipova et al. 2001; Burke et al. 2002). Detailed studies of $L 1$ elements in mammals (Hardies et al. 1986; Pascale et al. 1993; Casavant et al. 1996; 1998) and R1 and R2 elements in Drosophila (Lathe et al. 1995; Eickbush and Eickbush 1995; Eickbush et al. 1995; Lathe and Eickbush 1997) revealed that phylogenies of retrotransposons are generally consistent with species phylogenies and with the vertical inheritance/evolution of 
LINEs. There is however, evidence for infrequent horizontal transfer of non-LTR

retrotransposons between species (Kordis and Gubensek 1995, 1997, 1998; Sezutsu et al. 1995). Horizontally transferred elements and newly transposed LINEs presumably still encode enzymes necessary for further transposition and would be potentially mobile. Degenerate LINEs (reviewed in Smit 1996; Finnegan 1997) undoubtedly lost their ability to transpose either during or after integration into the genome.

Well-studied SINE families include $S 1$ in Brassiceae and Cruciferae (Gilbert et al. 1997; Lenoir et al. 1997), HpaI and SmaI in salmonids (Takasaki et al. 1994), SmaI-cor in corregonid fishes (Hamada et al. 1997), and CHRS, CHR1, and CHR2 in Cetaceans and Artiodactyls (Shimamura et al. 1999). Phylogenetic analysis of the S1 SINEs suggest that, as for LINEs, SINE evolution generally correlates with host species evolution. However, phylogenetic relationships among species and their transposable elements do not always agree, raising the possibility that elements are acquired by some species or individuals by horizontal transfer (Hamada et al. 1997). SINEs can be classified into subfamilies based on an analysis of diagnostic residues, i.e., bases differing from a consensus, putative ancestral sequence. Takasaki et al. (1994) noted the existence of members of the same HpaI SINE subfamilies "in different salmonid lineages and the amplification of members of different subfamilies in the same... lineage". This is consistent with the origin of such SINEs from multiple source genes, as opposed to a single "master" gene (Matera et al. 1990; Leeflang et al. 1992; Takasaki et al. 1994; 1996; 1997), or with horizontal transfer of the element between species. Horizontal transfer of a SmaI-cor SINE from corregonid fishes to salmonids has been suggested based on comparisons of intraspecific consensus sequences of the elements (Takasaki et al. 1997; Hamada et al. 1997). 
The mechanism of SINE mobility has been a matter of some speculation. Since SINEs lack genes encoding activities required for transposition, their mobility may depend on that of LINEs. During transposition, a LINE-encoded endonuclease nicks chromosomal DNA and an elementencoded reverse transcriptase directs reverse transcription of the cDNA copy from the cut site directly onto the target site (Luan et al. 1993; Burke et al. 1999). Similar target site duplications flanking primate $A l u$ and rodent $I D, B 1$ and $B 2$ SINEs as well as mammalian $L 1$ LINEs (Feng et al. 1996; Jurka and Klonowski 1996; Boeke 1997; Jurka 1997; Cost and Boeke 1998) suggest that the LINE enzyme may reverse transcribe and initiate the transposition of SINE sequences. In addition, common sequence motifs at the $3^{\prime}$ ends of some SINE and LINE pairs might produce common 3'-end secondary structures that prime the reverse transcription of both elements (Jurka and Klonowski 1996; Ohshima et al. 1996).

Chironomid mitochondrial genes, nuclear genes, and the LINE and SINE sequences reported here are a uniquely large data set allowing for studies of species and retrotransposon evolution and their impact on genome remodeling. In this study, overall similar evolutionary pathways seen in mitochondrial gene and LINE phylogenies support the monophyletic origin of LINEs. While a SINE phylogeny separates similar chironomid groups, several statistically robust phylogenetic anomalies were noted among the these elements. Based on diagnostic residue analysis, a few are members of subfamilies shared by distantly related species, suggesting their acquisition by horizontal transfer. Finally, sequence similarities near the 3' ends of the LINEs and SINEs studied here suggest that CTRT1-like SINEs and NLRCth1-like LINEs are cotransposing and co-evolving pairs in chironomids. 


\section{Materials and Methods}

DNA Extractions, Polymerase Chain Reactions (PCR) and Cloning. In addition to laboratory populations of $C$. thummi, specimens were obtained from natural populations (Fig. 1 with more detail in Guryev et al. 2001). DNA was extracted from pooled fresh or frozen larvae as described (Blinov et al. 1997) or fixed in ethanol or ethanol/acetic acid (Guryev et al. 2001). Primers 5'-AGCAGCTACAGGCCAATAAGTCTAC and 5'-ATACAGTGCTGTATCATCTGCGAA amplify a $500 \mathrm{nt}$ region encoding 3 highly conserved domains of the reverse transcriptase gene in ORF (open reading frame) 2 of LINE NLRCth1 (Blinov et al. 1993, 1997). Primers 5'-CGTTGGCCTTACGTGCCAAA and 5'-CTTCAGCTGGAGGCACTG amplify most of SINE CTRT-1 (Gruhl et al. 2000). For PCR product cloning, phosphorylated primers with 5' pentameric EcoRI linkers were synthesized in the State Research Center of Virology and Biotechnology "Vector”, Novosibirsk, Russia.

PCR-amplification was with thermal-stable DNA polymerases including Taq (Perkin-Elmer Applied Biosystems Division, Foster City, CA) and EnzyPlus polymerase (EnzyPol Ltd., Denver, CO) under standard conditions. SINE amplification was as described previously (Gruhl et al. 2000). Conditions for LINE NLRCth1 amplification were the same, except that the annealing temperature was increased from $42^{\circ} \mathrm{C}$ to $48^{\circ} \mathrm{C}$. Ethidium bromide $1 \%$ agarose gels were used to determine DNA concentrations. For cloning, PCR products made with EcoRI linker primers were gel-purified (Magic PCR Prep DNA Purification System, Promega, Madison, WI) and treated with T4 polymerase in the presence of dGTP to generate EcoRIcompatible cohesive ends (Aslanidis and de Jong 1990). The PCR products were ethanol- 
precipitated and redissolved in TE buffer; $50 \mathrm{ng}$ were ligated into $10 \mathrm{ng}$ of EcoRI-digested pBlueScript plasmid (Stratagene, La Jolla, CA). Recombinant plasmids were transformed into XL1-Blue cells (Stratagene). Screening was by blue-white selection. Plasmid DNA was extracted using the Wizard Plus Miniprep DNA Purification System (Promega, Madison, WI).

In preliminary studies of 51 geographically (Palearctic, nearctic, Australian, Oceanian and Southeast Asian) and five phyletically diverse Chironomidae genera (Chironomus, Kiefferulus, Glyptotendipes, Sergentia, Archaeochlus), NLRCthl copies could be amplified in the Chironomus (except for C. hawaiiensis), Kiefferulus, and Glyptotendipes genera. In contrast, CTRT1-like SINEs were amplified from 37 of 47 species of the genus Chironomus and were not detected in members of other chironomid genera. For this report, SINEs and LINEs were analyzed only in Palearctic (C. thummi, C. tentans, C. pallidivitattus, C. luridus, C. dorsalis, $C$. agilis, C. plumosus, C. annularius, C. cingulatus, C. balatonicus, C. tuvanicus), Australian (C. duplex, C. 'februarius', C. nepeanensis, C. tepperi, C. australis), one Southeast Asian (C. circumdatus) and one Oceanian (C. crassiforceps) species (the latter two species are also Australian, but separated by the continent).

DNA Sequencing and Analyses. Manual DNA sequencing (both directions) was done with the dsDNA Cycle Sequencing kit (Gibco BRL-Life Technologies, Gaithersburg, MD). Automated DNA sequencing (both directions) was done using $\mathrm{ABI}$ dye-linked terminators with an $\mathrm{ABI}$ 373A automated DNA sequencer (Perkin Elmer, Foster City, CA). DNA sequences were aligned manually with ESEE (Eyeball Sequence Editor, V.3.05, Eric Cabot, 1995) or FAR (v. 1.6, E. Roshal, 1996-1998). LINE and SINE sequence alignments are deposited in EMBL 
database under accession numbers ALIGN_000097 and ALIGN_000245, respectively; GenBank accession numbers for individual sequences in the alignments are linked with these ALIGN accession numbers. GenBank accession numbers for sequences of amplified but severely truncated LINEs not included in ALIGN_000097 are AF352405-AF352409 and AF352513AF352531. GenBank accession numbers for SINEs from five species not included in the ALIGN_000245 alignment are AF366370-AF366374 (elements from C. borokensis, $C$. muratensis, C. piger, C. pseudothummi and C. spilleri, respectively).

Since maximum parsimony and neighbor-joining (NJ) methods in the Mega 2.0 package (Kumar et al. 2000) generated similar phylogenetic trees for LINEs and for SINEs, only NJ results are reported here. Statistical support for the trees was evaluated by bootstrap analysis (1000 replications, Felsenstein 1985). With a few exceptions, bootstrap values of $<51 \%$ are not shown on phylogenetic trees. LINEs with interrupted ORF2 sequences (i.e., deletions or insertions that would result in inappropriate coding potential) were not included in the phylogenetic analyses. Severely truncated SINEs were also excluded from the phylogenies. Diagnostic residues were determined by the methods of several investigators (Deragon et al. 1994; Hamada et al. 1997; Takasaki et al. 1996; Kido et al. 1994,1995). A single consensus sequence was determined for all aligned SINEs using the "majority" rule of Kido et al. (1995); positions where no base exceeded $50 \%$ were assigned IUPAC standard mixed-base notations. Residues showing a systematic pattern of divergence from the consensus sequence are defined as diagnostic. To help define subfamilies of the element, an NJ tree was constructed with PAUP (Swofford 1993) using distances based on the absolute number of differences between the SINE diagnostic residues. 


\section{Results and Discussion}

LINE NLRCth1 and SINE CTRT1, originally discovered in C. thummi, have since been found in other chironomid species (Blinov et al. 1993; 1996; Gruhl et al. 1997; 2000). If both elements predate radiation of the chironomids, then both should be widespread in the genus. Therefore, the species studied here were chosen to represent a broad phylogenetic distribution (Fig. 1). In the absence of interspecific DNA exchange by introgression or "horizontal" transfer, gene phylogenies should be consistent with the species relationships that assume vertical descent from common ancestors. The promiscuous mobility of some transposable elements can confound the interpretation of molecular phylogenies because a) multicopy elements that are no longer mobile are not under selective pressure and should rapidly accumulate mutations, even within a species, and b) the potential of horizontal transfer can increase multicopy sequences. It is therefore necessary to analyze many copies of transposable elements from each species in order to establish meaningful evolutionary relationships. This was done for 306 copies of NLRCth1 LINEs and CTRT1 SINEs PCR-amplified and cloned from 18 chironomid species.

\section{Conservation of LINEs and SINEs.}

NLRCth1-like LINEs are similar to the original NLRCth1 (Blinov et al. 1993): $500 \mathrm{nt}$ long, strongly conserved, and with no premature stop codons in the ORF2 reverse transcriptase. Only a few LINEs (4, 3 and 5 from pallidivitattus, duplex, and circumdatus respectively; see EMBL Accession \# ALIGN_000097) had significant deletions in ORF2. Based on in situ hybridization of an NLRCth1 probe to salivary gland cell polytene chromosomes (Blinov et al. 1996), there are 
60-100 hybridization sites for species with low nucleotide divergence between LINEs, compared to about 20 sites for species with more highly diverged elements. This is consistent with a longer period of inactivity of the latter LINEs and/or with the transposition of fewer copies of these elements.

To estimate selective pressure on the ORF2 reverse transcriptase region in the different species, ratios of synonymous to non-synonymous base substitutions (Ks/Ka) were calculated by aligning a consensus sequence of $C$. nepeanensis (the most diverged species; Fig. 1,2) with individual full length LINEs (EMBL Accession \# ALIGN_000097) or with LINE consensus sequences for the other species. Since there was no significant difference in Ks/Ka values comparing individual or consensus sequences, only the latter are reported here. Ks/Ka ratios ranged from 6.1 to 10.7 . This is within or even higher than the $\mathrm{Ks} / \mathrm{Ka}$ range of 1.78 to 8.5 determined for the chironomid globin $2 \beta$ gene (alignment not shown). Apparently, comparable selective pressure is maintaining active LINEs in the different species. Like chironomid LINEs, 41 apparently intact Rte-1 elements in Caenorhabditis elegans had high $\mathrm{Ks} / \mathrm{Ka}$ ratios indicating selective pressure to preserve the continued mobility of most of the elements. On the other hand, lower Ks/Ka ratios for 9 deleted, more diverged Rte-1 elements (Malik and Eickbush 1998), indicating that the loss of reverse transcriptase function permanently disables the element. The loss of mobility is then followed by more rapid sequence divergence and an increase in the rate of non-synonymous base substitutions. When Chironomus LINEs with major deletions were included in $\mathrm{Ks} / \mathrm{Ka}$ calculations (data not shown), the ratios were comparable to undeleted LINEs, indicating that the shortened LINEs must have retroposed recently before being inactivated by deletion. 
As SINEs do not contain ORFs, Ks/Ka values do not apply. Alignment of SINEs from 146 clones representing 22 species generates 399 residues (EMBL Accession \# ALIGN_000245). Scattered small indels characteristic of a few elements are most likely insertions; substantially deleted elements were found in C. tuvanicus (tuv2-tuv7), C. crassiforceps (cra3-cra9) and C. agilis (agi2-agi8, agil1). Nevertheless, 303 of the 399 positions in the alignment are $\geq 80 \%$ identical. This relatively high level of sequence conservation of otherwise non-selected SINE copies is consistent with the recent mobility of a CTRT1-like source SINE(s) within each species.

\section{Phylogenetic analyses and the construction of evolutionary trees}

A comprehensive study of mitochondrial genes (Fig.1) defines phylogenetic groups containing the 'ancient' Australian species (cluster I), 'modern’ Australian species (cluster II), Camptochironomids (cluster III), 'pseudothummi' clade (cluster IV) and 'plumosus' phylogenetic group (cluster V). The topology of the NLRCth1 LINE tree follows that of the mitochondrial tree (Guryev et al. 2001, Fig.2), both showing C. nepeanensis to be the most diverged (i.e. ancient) species. This is similar to the congruence $R 1$ and $R 2$ LINE families in Drosophila with molecular as well as morphological phylogenies (Lathe et al. 1995; Eickbush and Eickbush 1995; Eickbush et al. 1995; Lathe and Eickbush 1997). Given the close phylogenetic relationship between C. plumosus, balatonicus and agilis, fortuitous convergence at key residues may explain the misplacement of the plu2 and plu6 LINEs with balatonicus and of plu8 with agilis elements (boxed in Fig. 2).

Most SINEs separate by species in the SINE phylogeny, and C. nepeanensis SINEs remain the 
longest diverged lineage. However, in all other respects, the SINE tree topology differs from that of the mitochondrial and LINE trees, indicating that SINE evolution does not track species evolution. In addition, many more SINEs than LINEs are cladistically misplaced (Fig. 3). Albeit with slim bootstrap support (41\%), the dorsalis SINEs are on a peripheral branch in the same clade containing the Australian and 'plumosus' group species. Other misplaced elements with weak support ( $<51 \%$ bootstrap values) include: plu2 and plu9 in clades that contain balatonicus elements; bal3 lying by itself and basal to all of the other plumosus, agilis and balatonicus elements; ann 1 lying in the cingulatus clade; dup11 lying in a clade that includes agi6, both separated from other agilis elements; aus5 lying basal to separate clades containing duplex and other australis elements; dup14 appearing more ancient than other duplex elements; pal4 branching with cingulatus. The luridus SINEs comprise a clade incorrectly separated from its pseudothummi group (IV) relatives, but with relatively strong (70\%) bootstrap support. And the clade including luridus and the more distantly related tentans elements is even more strongly supported (70\% bootstrap). Even though pallidivitattus and dorsalis are not closely related, pall and pal5 branch with dorsalis elements with surprisingly strong (90\%) bootstrap support. Excluding unlikely errors in species identification, these cladistic misplacements can be explained by a) random base substitutions resulting in sequence convergence of elements in different species, b) the maintenance of source elements present at divergence by different species, or c) the horizontal transfer of either source SINEs or SINE copies between species. Since more SINE than LINE misplacements occur, and since some of these involve distantly related species with robust bootstrap support, alternative methods were sought to resolve the SINE anomalies. 


\section{Determination of SINE subfamilies}

Percent similarity data were generated for both LINEs and SINEs (data not shown). As has been shown for other elements (e.g., Lathe et al. 1995; McAllister and Werren 1997; Marin et al. 1998; Hamada et al. 1997; Lenoir et al. 1997), the data suggest subfamilies generally consistent with cladistic groupings in the $\mathrm{NJ}$ phylogenies reported here, but did not shed light on the anomalous placement of many elements in the SINE phylogeny. Therefore, diagnostic residues were determined in the hope of clarifying subfamily relationships of the SINEs. The assumption of this analysis is that active multiple source SINEs diverge from their common ancestor at different residues before transposition. After transposition, source elements are distinguished by the subfamily similarity of their copies. Related species should share SINE copies belonging to the same or a similar SINE subfamily, while more diverged species should not share many similar diagnostic residues. A single species may also harbor copy SINEs belonging to more than one subfamily if it contains one or more divergent source elements.

As expected, SINE diagnostic residue patterns (Fig. 4) show that phylogenetically related species share many diagnostic residues, defining more inclusive subfamilies of CTRTI-like elements. The latter could arguably be further subdivided. Several species do in fact contain more than one SINE subfamily. These include e.g., tuv1 and tuv2 vs the other tuv elements; thu 10 and thu 2 s each other and the remaining thu elements; agi7 $v s$ other agi elements; bal3, 10 and $12 v s$ other bal elements. The grouping of diagnostic residues in Fig. 4 optimizes some but obscures other relationships (e.g., among Australian species). An unrooted NJ cladogram of aligned diagnostic residues more clearly resolves eight major subfamilies (Fig. 5). That the consensus sequence reflects a putative ancestral SINE is supported by its basal position in the 
tree. Compared to the SINE phylogeny, differences in the diagnostic residue cladogram include: 1) the previously misplaced annl now lies with most of other annularius elements in a polytomous clade, 2) dup14 now groups with australis elements instead of other duplex SINEs; 3) dup11 grouped with crassiforceps SINEs instead of with agilis SINEs; 4) bal3 is newly joined by five agilis elements. Clearly, the tree building algorithms 'see' actual sequences differently from the more derivative diagnostic residue alignments. Nevertheless, the pal4, pal1, pal5, plu2, plu9 are similarly "misplaced" on the diagnostic residue cladogram and SINE phylogeny, and lur elements still group apart from their pseudothummi relatives. Several elements not originally included in the SINE phylogeny were included in building the consensus sequence for diagnostic residue analysis. Among these, feb6, feb11, thu10, ballo, and are also misplaced in the diagnostic residue cladogram. Among SINEs that are the sole representatives of their species, pigl (from C. piger) shares diagnostic residues with SINEs of thummi, its sibling species and murl and borl SINEs group with their plumosus group relatives. On the other hand, psel and lur1-6, though from related species, are separated from their thummi relatives, and the Australian spil species is misplaced among the plumosus group elements.

Are SINEs from related species that share diagnostic residues and branch incorrectly with those of the relative (e.g., plu2 and plu9 in the balatonicus clade, ann1 in the cingulatus clade) due to horizontal transfer? Since theses cases are only weakly supported by bootstrapping, could they be artifacts of convergent base changes at one or two diagnostic residues that tree building algorithms treat as informative. In contrast, a horizontal transfer mechanism might best explain the robust support for separation of luridus from other pseudothummi group SINEs, and for the cingulatus-like (pal4) and dorsalis-like (pal1, pal5) SINEs. If so, how and when did the transfer 
occur between these phylogenetically distant species? Alternatively, were all SINE subfamilies present before most speciation and then inherited linearly, with retention or silencing of some source elements in some species? These questions remain to be resolved.

\section{Chironomid SINE and LINE co-evolution and origins}

LINEs can exist without SINEs, e.g., in C. elegans, D. melanogaster (C. elegans Sequencing Consortium 1998; Adams et al. 2000), but so far, not vice versa. Since SINEs do not encode enzymes needed for mobility, LINEs may provide enzymes for SINE transposition (Jurka and Klonowski 1996; Ohshima et al. 1996; Jurka 1997; Okada et al. 1997; Malik and Eickbush, 1998). We note that no C. tuvanicus or C. crassiforceps LINEs were clonable from PCR product smears eluted from gels, and all tuvanicus and crassiforceps SINEs (except tuv1, cra1 and cra2) are significantly deleted. Conversely, C. agilis LINEs seem intact while its SINEs are largely deleted, suggesting that the source SINE has been dormant for a long time, perhaps even during a recent mobilization of the LINE.

Homologous motifs at the 3'-end sequences of SINEs and LINES may provide a mechanism for the common priming reverse transcription of the RNAs of both elements into mobile DNAs (Ohshima et al. 1996). Similar 3' motifs are known for >10 pairs of elements from tortoise, turtle, fish, mammals, and plants (Smit 1996; Kajikawa, Ohshima, and Okada 1997;Okada et al. 1997; Okada and Hamada 1997; Terai et al. 1998; Gilbert and Labuda 1999; Ogiwara et al. 1999). A similarity between 3 ' motifs of MIR SINEs and the $C R 1$ LINE even hint that some ancient SINE/LINE pairs evolved before separation of mammalian and reptilian lineages

(Ohshima et al. 1996; Okada et al. 1997; Gilbert and Labuda, 1999). In C. thummi, the original 
genomic clones of LINE NLRCth1 and SINE CTRT1 share >45\% similarity over a 64 nucleotide region near their 3'-ends (Fig. 6), supporting a hypothetical, co-evolving NLRCth1/CTRT1 pair in which the mobility of the SINE depends on that of the LINE.

If SINE transposition depends on LINE mobility, then LINEs must have evolved first. A modern classification of LINEs includes more than 70 eukaryotic elements divided into twelve families (Malik et al. 1999; Malik and Eickbush, 1999). In this classification all LINEs have a common root in the $\mathrm{Cre}$ clade, an ancient LINE distributed only among primitive eukaryotes.

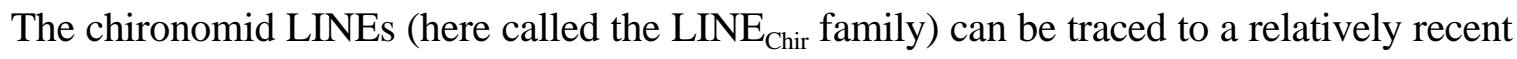
branch of these elements. Members of the $\operatorname{LINE}_{\text {Chir }}$ family were amplified from the related Kiefferulus and Glyptotendipes genera (this study), and are even older than the family Chironomidae (which include all chironomid genera), being structurally related to the juan family of elements in the jockey clade (Malik et al. 1999) widely distributed in Diptera.

Because SINE families show little or no sequence similarity across phyla, deep rooted tree construction is not possible. SINEs do not have a common origin and probably appeared independently in different taxa. Among Chironomidae, only the genus Chironomus contain CTRT1-like SINEs (the $\operatorname{SINE}_{\text {Chir }}$ family). Within this genus, SINE amplification failed in $C$. hawaiiensis, C. sp3 (Cook Islands, Oceania), C. sp2 (Rio de Janeiro, South America), C. xanthus (South America), C. cucini, C. tigris, C. entis, C.decorus group sp1 (North America), C. tenuistylus and Lobochironomus sp. (Palearctic). This suggests that the $\mathrm{SINE}_{\text {Chir }}$ family of elements (including CTRTI) originated in an ancestor common to the Australian and Palearctic species. 
Summarizing evolutionary data (Makarevich et al, 2000; Guryev et al, 2001, this report), the common ancestor to the genus Chironomus (already containing an NLRCthl-like LINE) must have appeared in Pangaea, before drift separated present day continents about 120 million years ago. Among the species studied, the most ancient are South American. Species migrating to Australia via the Antarctic region would have established the northern and southern Australian phylogenetic groups, and already would have contained CTRT1-like SINEs. Moving further north to Asia and then Europe, the northern Australian group would then have established the Palearctic lineages, including the phylogenetically recent camptochironomid, pseudothummi and plumosus groups. Representatives of the latter groups then migrated across the Bering Straight to North America to establish the North American nearctic species. Based on their outlying positions on the mitochondrial gene phylogeny, their intronless $2 \beta$ globin genes (data not shown) and their lack of a PCR-amplifiable CTRTI-like SINE, other North American species such as C. cuccini and C. tigris most likely moved north from South America some time before another South American species began its/their antarctic migration. Assuming Africa to be a side trip during migration to Australia, it will be of great interest to collect data from African chironomids to will place them in their correct evolutionary context and pinpoint more accurately the time of origin of CTRT1-like SINEs.

We began this study of $\operatorname{LINE}_{\text {Chir }}$ and $\mathrm{SINE}_{\text {Chir }}$ retrotransposons with two questions. 1) Does retroelement evolution correlate with that of the host species? 2) Do the LINEs and SINEs co-

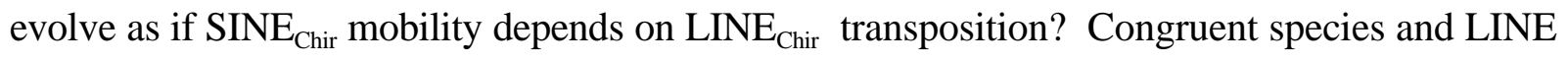
phylogenies indicate that LINEs have been inherited by linear descent. The non-congruence of the SINE tree topology with other phylogenies indicates an expected rapid divergence of copy 
SINEs in the absence of selection, while the tendency of most SINEs from the same species to group together supports their independent and relatively recent mobilization in different species. While convergent base changes may explain many cladistically misplaced SINEs (and a few LINEs), others in distantly related species may have been shared by horizontal transfer or are ancient SINE subfamilies retained in some but lost in other species. Finally, the fact that SINEs and LINEs retain 3'-motifs of $>45 \%$ similarity, and that degenerate LINEs accompany degenerate SINEs in a few species are circumstantial evidence for chironomid LINE and SINE co-evolution, and the dependence of SINE on LINE mobility.

Acknowledgments. The study was supported by Russian Foundation for Basic Research Grant 99-04-49265 and by the University of Wisconsin-Milwaukee. We thank Dr. K. Aimanova, Dr. A. Istomina, and Dr. L. Gunderina for collection and karyological identification of Palearctic chironomids, Drs. G. Strixino, K. Kawai, O. Leoncini and L. Doig and M. Nishimoto for collection of South American and Oceania chironomids and Dr. M. Butler for providing $C$. decorus group sp 1, C. cucini, and C. 'tigris'. 


\section{References}

Adams MD, Celniker SE, Holt RA, Evans CA, Gocayne JD et. al. (2000) The Genome Sequence of Drosophila melanogaster. Science 287:2185-2195

Archipova IR, Morrison HG (2001) Three retrotransposon families in the genome of Giardia lamblia: two telomeric, one dead. PNAS 98:14497-14502

Aslanidis C, de Jong PJ (1990) Ligation-independent cloning of PCR products (LIC-PCR). Nucleic Acids Res. 18:6069-6074

Bennetzen JL (2000) Transposable element contributions to plant gene and genome evolution. Plant. Mol. Biol. 42:251-269

Blinov AG, Sobanov YV, Bogachev SS, Donchenko AP, Filippova, MA (1993) The Chironomus thummi genome contains a non-LTR retrotransposon. Mol. Gen. Genet. 237: $412-420$

Blinov AG, Scherbik SV, Kiknadze II, Filippova MA, Aimanova, KG (1996) Distribution of the NLRCth1 non-LTR retrotransposon is restricted to the genus Chironomus. Genetika (in Russian) 32:1616-1622

Blinov AG, Sobanov YV, Scherbik SV, Aimanova, KG (1997) The Chironomus (Camptochironomus) tentans genome contains two non-LTR retrotransposons. Genome $40: 143-150$

Boeke JD 1997. LINEs and Alus - the polyA connection. Nat. Genet. 16:6-7

Burke WD, Malik HS, Jones JP, Eickbush TH (1999) Conserved structure and mechanism of integration of the R2 retrotransposable element in all arthropods. Mol. Biol. Evol. 16:502511 
Burke WD, Malik HS, Rich SM, Eickbush TH (2002) Ancient lineages of non-LTR

retrotransposons in the primitive eukaryote, Giardia lamblia. Mol Biol Evol, 19: 619-630

Britten RJ (1997) Mobile elements inserted in the distant past have taken on important functions.

Gene 205:177-182

Casavant NC, Sherman AN, Wichman HA(1996) Two persistent LINE-1 lineages in

Peromyscus have unequal rates of evolution. Genetics 142:1289-1298

Casavant NC, Lee RN, Sherman AN, Wichman HA (1998) Molecular evolution of two lineages of L1 (LINE-1) retrotransposons in the california mouse, Peromyscus californicus. Genetics $150: 345-357$

C. elegans Sequencing Consortium. (1998) Genome sequence of the nematode C. elegans: a platform for investigating biology. Science. 282:2012-2018

Cost GJ, Boeke JD (1998) Targeting of human retrotransposon integration is directed by the specificity of the $L 1$ endonuclease for regions of unusual DNA structure. Biochemistry $37: 18081-18093$

Deragon JM, Landry BS, Pelissier T, Tutois S, Tourmente S, Picard G (1994) An analysis of retroposition in plants based on a family of SINEs from Brassica napus. J. Mol. Evol. $39: 378-386$

Eickbush DG, Eickbush TH (1995) Vertical transmission of the retrotransposable elements $R 1$ and $R 2$ during the evolution of the Drosophila melanogaster species subgroup. Genetics $139: 671-684$

Eickbush DG, Lathe III WC, Francino MP, Eickbush TH (1995) $R 1$ and $R 2$ retrotransposable elements of Drosophila evolve at rates similar to that of nuclear genes. Genetics 139:685695 
Felsenstein J (1985) Confidence limits on phylogenies: an approach using the bootstrap. Evolution 39:783-791

Feng Q, Moran JV, Kazazian HH, Boeke JD (1996) Human L1 retrotransposon encodes a conserved endonuclease required for retrotransposition. Cell 87:905-916

Finnegan DJ (1997) Transposable elements: how non-LTR retrotransposons do it. Cur. Biol. 7:R245-R248

Gilbert N, Arnaud P, Lenoir A, Warwick SI, Picard G, Deragon JM (1997) Plant S1 SINEs: a model to study retroposition. Genetica 100:155-160

Gilbert N, Labuda, D (1999) CORE-SINEs: eukaryotic short interspersed retroposing elements with with common sequence motifs. Proc. Natl. Acad. Sci. USA 96:2869-2874

Gruhl M. C., Kao W-Y, Bergtrom G (1997) Evolution of orthologous intronless and intronbearing globin genes in two insect species. J. Mol. Evol. 45:499-508

Gruhl MC, Scherbik SV, Aimanova KG, Blinov A, Diez J-L, Bergtrom G (2000) Insect globin gene polymorphisms: intronic minisatellites and a retroposon interrupting exon I of homologous globin genes in Chironomus (Diptera). Gene. 251:153-163

Guryev V, Makarevitch I, Blinov A, Martin J (2001) Phylogeny of the genus Chironomus (Diptera) inferred from DNA sequences of mitohondrial cytochrome $b$ and cytochrome oxidase I. Mol. Phyl. Evol. 19:9-21

Hamada M, Kido Y, Himberg M, Reist JD, Ying C, Hasegava M, Okada.N (1997) A newly isolated family of short interspersed repetitive elements (SINEs) in corregonid fishes (whitefish) with sequences that are almost identical to those of the SmaI family of repeats: possible evidence for the horizontal transfer of SINEs. Genetics 146:355-367 
Hardies SC, Martin SL, Voliva CF, Hutchison CA, Edgell MH (1986) An analysis of replacement and synonymous changes in the rodent L1 repeat family. Mol Biol Evol. $3: 109-125$

Jurka J, Klonowski P (1996) Integration of transposable elements in mammals: selection of target sites. J. Mol. Evol. 43:685-689

Jurka J (1997) Sequence patterns indicate an enzymatic involvement in integration of mammalian retroposons. Proc. Natl. Acad. Sci. USA 94:1872-1877

Kajikawa M, Ohshima K, Okada N. (1997) Determination of the entire sequence of turtle CRl: the first open reading frame of the turtle $C R l$ element encodes a protein with a novel zinc finger motif. Mol. Biol. Evol. 14:1206-1217

Kazazian H, Moran J (1998) The impact of $L 1$ retrotransposons on the human genome. Nature Genet. 19:19-24

Kido Y, Himberg M, Takasaki N, Okada N (1994) Amplification of distinct subfamilies of short interspersed elements during evolution of the Salmonidae. J. Mol. Biol. 241:633-644

Kido Y, Saitoh M, Murata S, Okada N (1995) Evolution of the active sequences of the HpaI short interspersed elements. J. Mol. Evol. 41:986-995

Kidwell MG, Lisch DR (2000) Transposable elements and host genome evolution. Trends Ecol. Evol. 15:95-99

Kordis D, Gubensek F (1995) Horizontal SINE transfer between vertebrate classes. Nat. Genet. $10: 131-132$

Kordis D, Gubensek F (1997) Bov-B long interspersed repeated DNA (LINE) sequences are present in Vipera ammodytes phospholipase $A 2$ genes and in genomes of Viperidae snakes. Eur. J. Biochem. 246:772-779 
Kordis D, Gubensek F (1998) Unusual horizontal transfer of a long interspersed nuclear element between distant vertebrate classes. Proc. Natl. Acad. Sci. U S A. 95:10704-10709

Kumar S., K. Tamura , I. B. Jakobsen, M. Nei. (2000) MEGA: Molecular Evolutionary Genetics Analysis, Version 2.0. Pennsylvania State University, University Park, and Arizona State University, Tempe

Lathe WC III, Burke WD, Eickbush DG, Eickbush TH (1995) Evolutionary stability of the $R I$ retrotransposable element in the genus Drosophila. Mol. Biol. Evol. 12:1094-1105

Lathe WC III, Eickbush TH (1997) A single lineage of $R 2$ retrotransposable elements is an active, evolutionary stable component of Drosophila rDNA locus. Mol. Biol. Evol. 14:12321241

Leeflang EP, LiU W-M., Hashimoto C, Choudary PV, Schmid, CW (1992) Phylogenetic evidence for multiple Alu source genes. J. Mol. Evol. 37:559-565

Lenoir A, Cournoyer B, Warwick S, Picard G, Deragon JM (1997) Evolution of SINE S1 retroposons in Cruciferae plant species. Mol. Biol. Evol. 14:934-941

Linderberg B, Wiederholm T (1979) Notes on the taxonomy of European species of Chironomus (Diptera: Chironomidae). Ent. Scand. Suppl. 10:99-116

Lovsin N, Gubensek F, Kordis D (2001) Evolutionary Dynamics in a novel L2 clade of non-LTR retrotransposons in Deuterostomia. Mol Biol Evol, 18:2213-2224

Luan DD, Korman MH, Jakubczak JL, Eickbush TH (1993) Reverse transcription of R2Bm RNA is primed by a nick at the chromosomal target site: a mechanism for non-LTR retrotransposition. Cell 72:595-605

McAllister BF, Werren JH (1997) Hybrid origin of a B chromosome (PSR) in the parasitic wasp Nasonia vitripennis. Chromosoma 106:243-253 
Makarevich, IF, Berezikov EV, Guryev VP, Blinov AG (2000) Molecular phylogeny of the Chironomus genus deduced from nucleotide sequences of two nuclear genes, ssp160 and the globin $2 \beta$ gene. Molecular Biology. 34:606-612. Translated from Molekulyarnaya Biologiya, 34:701-707 (Russian)

Malik HS, Eickbush TH (1998) The RTE class of non-LTR retrotransposons is widely distributed in animals and is the origin of many SINEs. Mol. Biol. Evol. 15:1123-1134

Malik HS, Eickbush TH.(1999) NeSL-1, an ancient lineage of site-specific non-LTR retrotransposons from Caenorhabditis elegans. Genetics 154:193-203

Malik HS, Burke WD, Eickbush TH (1999) The age and evolution of Non-LTR retrotransposable elements. Mol. Biol. Evol. 16:793-805

Marin I, Plata-Rengifo P, Labrador M, Fontdevila A (1998) Evolutionary relationships among the members of an ancient class of non-LTR retrotransposons found in the nematode Caenorhabditis elegans. Mol. Biol. Evol. 15:1390-1402

Matera AG, Hellmann U, Hintz MF, Schmid CW (1990) Recently transposed Alu repeats result from multiple source genes. Nucl. Acids Res. 18:6019-6023

Ogiwara I, Masaki M, Ohshima K, Okada N (1999) Retropositional parasitism of SINEs on LINEs: identification of SINEs and LINEs in elasmobranchs. Mol. Biol. Evol. 16:1238-1250

Ohshima K, Hamada M, Terai Y, Okada N (1996) The 3'ends of the tRNA-derived short interspersed repetitive elements are derived from the 3'ends of long interspersed repetitive elements. Mol. Cell. Biol. 16:3756-3764

Okada N, Hamada M (1997) The 3' ends of tRNA-derived SINEs originated from the 3' ends of LINEs: a new example from the bovine genome. J. Mol. Evol. 44:52-56 
Okada N, Hamada M, Ogiwara I, Ohshima K (1997) SINEs and LINEs share common 3' sequences: a review. Gene 205:229-243

Pascale E, Liu C, Valle E, Usdin K, Furano AV (1993) The evolution of long interspersed repeated DNA (L1, LINE 1) as revealed by the analysis of an ancient rodent $L 1$ DNA family. J. Mol. Evol. 36:9-20

Sezutsu H, Nitasaka E, Yamazaki T (1995) Evolution of the LINE-like I element in the Drosophila melamogaster species subgroup. Mol. Gen. Genet. 249:168-178

Shimamura M, Abe H, Nikaido M, Ohima K, Okada N (1999) Geneaology of families of SINEs in Cetaceans and Artiodactyls: the presence of a huge superfamily of tRNA ${ }^{\text {Glu }}$ derived families of SINEs. Mol. Biol. Evol. 16:1046-1060

Smit AFA(1996) The origin of interpersed repeats in the human genome. Curr. Opin. Dev. $6: 743-748$

Swofford DL (1998) PAUP* 4.0 beta version 8 Phylogenetic Analysis Using Parsimony. Sinauer Associates Inc., Sunderland, MA

Takasaki N, Murata S, Saiton M, Kobayashi T, Park L, Okada N (1994) Species- specific amplification of tRNA-derived short interspersed repetitive elements (SINEs) by retroposition: a process of parasitization of entire genomes during the evolution of salmonids. Proc. Natl. Acad. Sci. USA, 91:10153-10157

Takasaki N, Park L, Kaeriyama M, Gharrett AJ, Okada N (1996) Characterization of speciesspecifically amplified SINEs in three salmonid species - chum salmon, pink salmon, and kokanee: the local environment of the genome may be important for the generation of a dominant source gene at a newly retroposed locus. J. Mol. Evol. 42:103-116 
Takasaki N, Yamaki T, Hamada M, Park L, Okada N (1997) The salmon SmaI family of short interspersed repetitive elements (SINEs): interspecific and intraspecific variation of the insertion of SINEs in the genomes of chum and pink salmon. Genetics 146:369-380

Terai Y, Takahashi K, Okada N (1998) SINE cousins: the 3'-end tails of the two oldest and distantly related families of SINEs are descended from the 3' ends of LINEs with the same genealogical origin. Mol. Biol. Evol. 15:1460-1471

Volff J-N, Korting C, Schartl M (2000) Multiple lineages of the non-LTR retrotransposons Rex1 with varying success in invading fish genomes. Mol Biol Evol, 17:1673-1684

Xiong Y, Eickbush TH (1990) Origin and evolution of retroelements based upon their reverse transcriptase sequences. EMBO J. 9:3353-3362 


\section{Figure Legends}

Fig. 1. Phylogenetic relationshsips of Chironomus species and list of chironomids used in the analysis. The majority-rule consensus neighbor-joining (NJ) tree based on sequences of mitochondrial genes $C O I$ and $C y t b$ and division of species into clusters I-VI is essentially as inferred in Guryev et al. (2001). Distantly related chironomid genera (Sergentia, Archaeochlus) and Drosophila are represented; species absent from the mitochondrial tree but used in the present study are listed below the tree. Plus and minus signs indicate positive and negative results of PCR amplification of NLR or SINE elements in a respective species. PCR products which have been cloned and sequenced are indicated by a circled + . Species for sequencing were chosen so that representatives from phylogenetic clusters I, II, III, IV and V are represented in the analysis. Geographical regions are: A - Australia, Af - Africa, PA - Palearctic, NA - North America, SA - South America, O - Oceania, SEA - South-East Asia. Scale is genetic distance as nucleotide (nt) divergence.

Fig. 2. Neighbor-joining tree of NLRCth1-like elements based on nucleotide sequences. Multiple cloned PCR-amplified products of a region of the reverse transcriptase open reading frame (ORF2; see text) were sequenced for each species. Clone names are derived from the first three letters of the respective species name followed by a clone number. Clones with identical sequences are represented by a single branch on the tree and clone numbers are separated by commas. Numbers at the nodes represent bootstrap values as percentage of 1000 replicates. Clones with unusual positions on the tree are boxed and discussed in the text. Scale is genetic distance (nt divergence). 
Fig. 3. Neighbor-joining tree of CTRT1-like SINE elements. Multiple cloned PCR-amplified products of a region within the SINE were sequenced for each species. Clone names and bootstrap values are as described for Fig. 2. Clones with unusual positions on the tree are boxed and discussed in the text. Scale is genetic distance (nucleotide divergence).

Fig. 4. Diagnostic Residues of CTRT1-like SINEs. Among the 146 aligned SINEs, 105 out of 399 positions were identified that contained bases differing from the consensus sequence of all SINEs (upper and lower case bases). Differences from the consensus due to transition as well as transversion were scored because the patterns generated by both represent data that should not be discounted. Diagnostic residues (uppercase boldface bases) were determined as described in the text. The alignment groups the diagnostic residues to optimize species relationships and putative SINE subfamilies in a maximum of species. Residue numbers for each base, and the consensus (Cons) base for each residue are repeated at the top and bottom of the alignment. Dots indicate the same base as the consensus, dashes are indels and ? indicates missing data. An asterisk (*) indicates the position of an 11 base insert (seen only the nep7B element) that was removed before analysis.

Fig. 5. Neighbor-joining unrooted phylogram of diagnostic SINE CTRT1 residues. The tree reveals 8eparated SINE subfamilies (numbered at right). It is possible to make finer subfamily distinctions based on clade separation, and several SINEs remain or are newly misplaced on this tree (cf. Fig. 3). 
Fig. 6. Similarities of aligned 3' ends of NLRCth1 from C. thummi and SINE CTRT1 from C. thummi. A 64 nucleotide region showing similarity between the elements is underlined; LINE vs SINE nucleotide identities are in boldface. Sequences are taken from: NLRCth1 (GeneBank Accession No. S59870) and CTRT1 (GenBank Accession No. AF001292). 


\section{FIGURE 1}

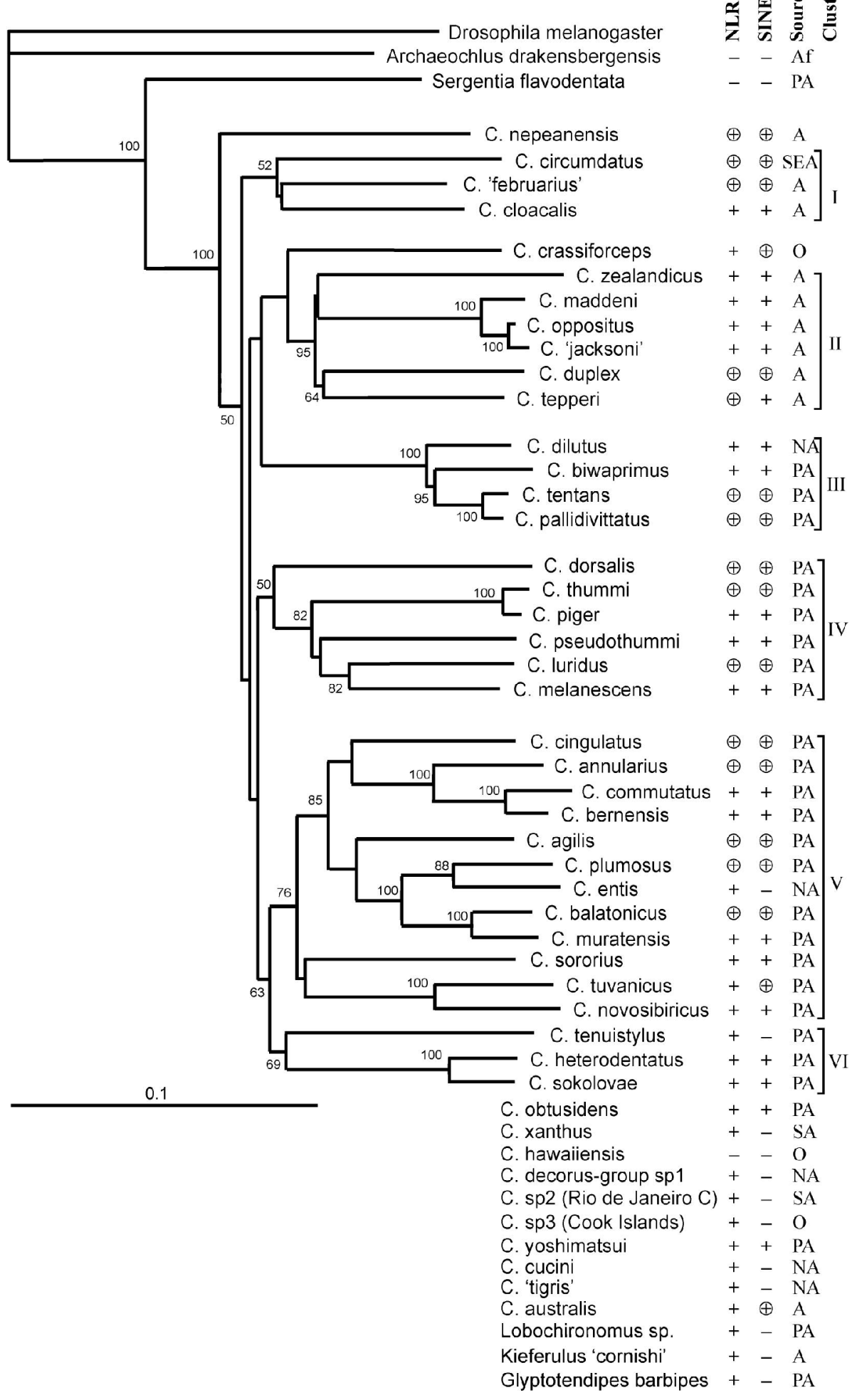




\section{FIGURE 2}

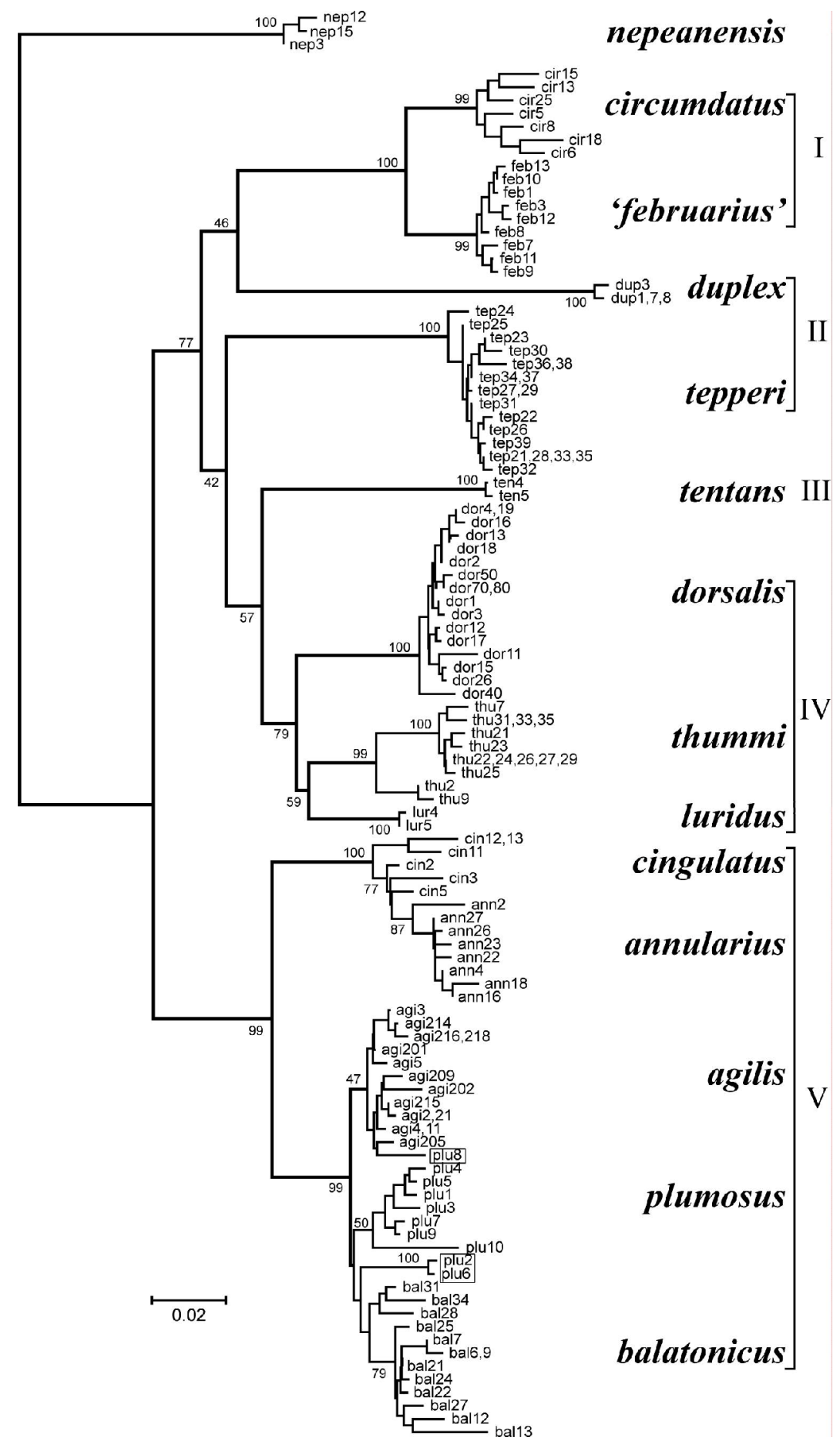




\section{FIGURE 3}

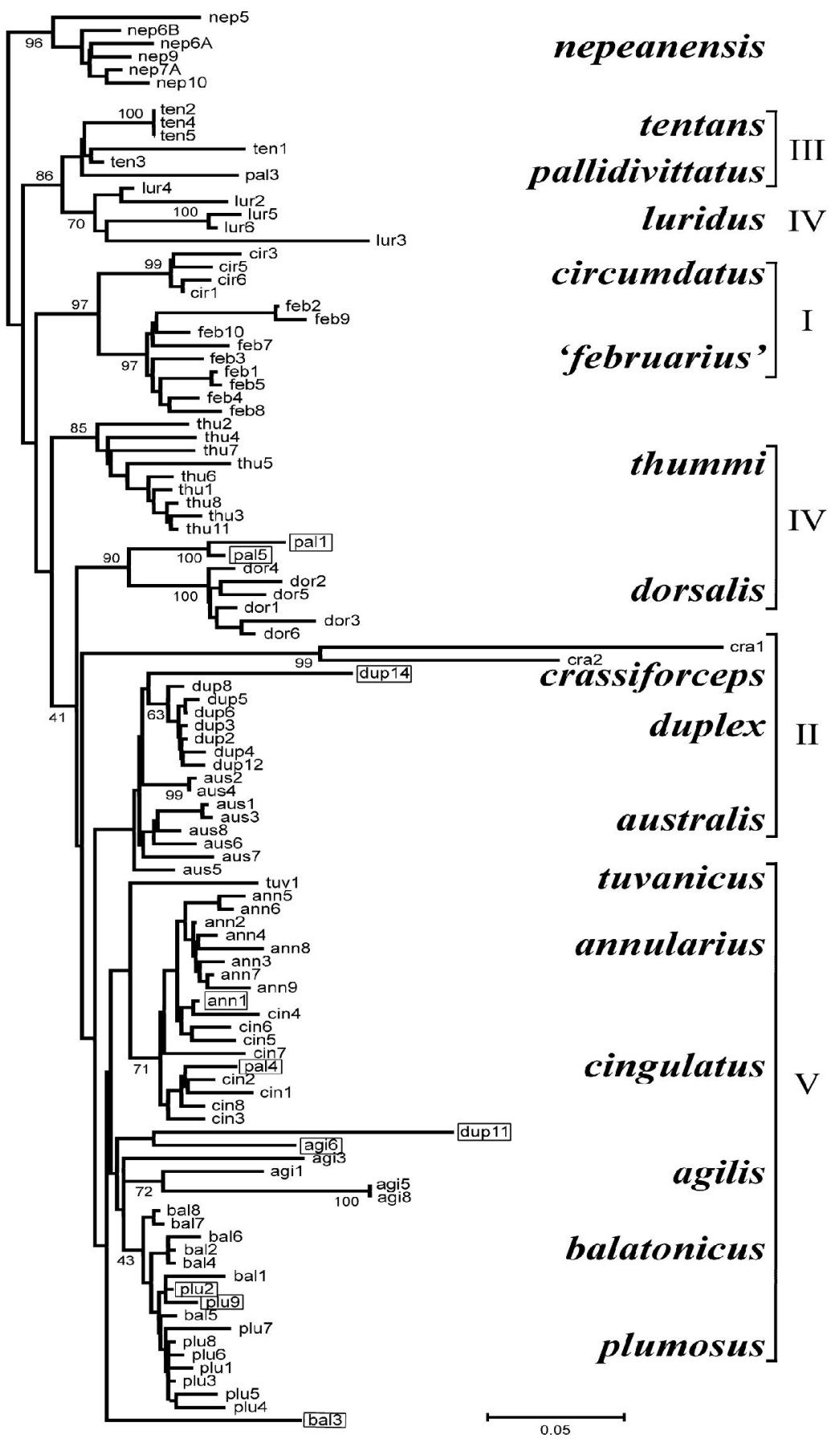




\section{FIGURE 4, p. 1}

$\begin{array}{lllllllllllll}223333333333 & 3333 & 2 & 211333333332213 & 21 & 122233311121 & 111111111 & 2 & 32231221 & 2122211331 & 21 & 2 & 2\end{array}$ 11245663344444555552761233389835888888890939333349077776227482477778288838951091232451104539744705821141

Cons

tuv1

tuv2

$\operatorname{tuv} 3$
$\operatorname{tuv} 4$

tuv5

tuv 6
tuv 7

dor 1

dor2

dor5

dor 4

dor 3

thu3

thu6
thul1

thus

thu 7

thul

thu 4
thus

thu2

thu10

lur

lur

$\operatorname{lur} 6$
$\operatorname{lur} 5$

$\operatorname{lur} 5$
$\operatorname{lur} 3$

lur2

ten 1

ten 4
ten 5

ten 3

pal1

pal2

pal5

pal4

cin1

$\operatorname{cin} 2$

cin6

cin8

cin 4

cins

ann1

ann2

ann7
ann8

ann 3

ann 9

ann5

ann6

plus

plus

plu5

plu6

plu10

plu2

plu9
plu7 GGACCTCTAG--------TGAGGC---GTCAA-------TCTATAACCTTATTTGGGAAATA----AC-AGCCgAtGCGGCCATGC-ATAT-GA-GCGGTCTT

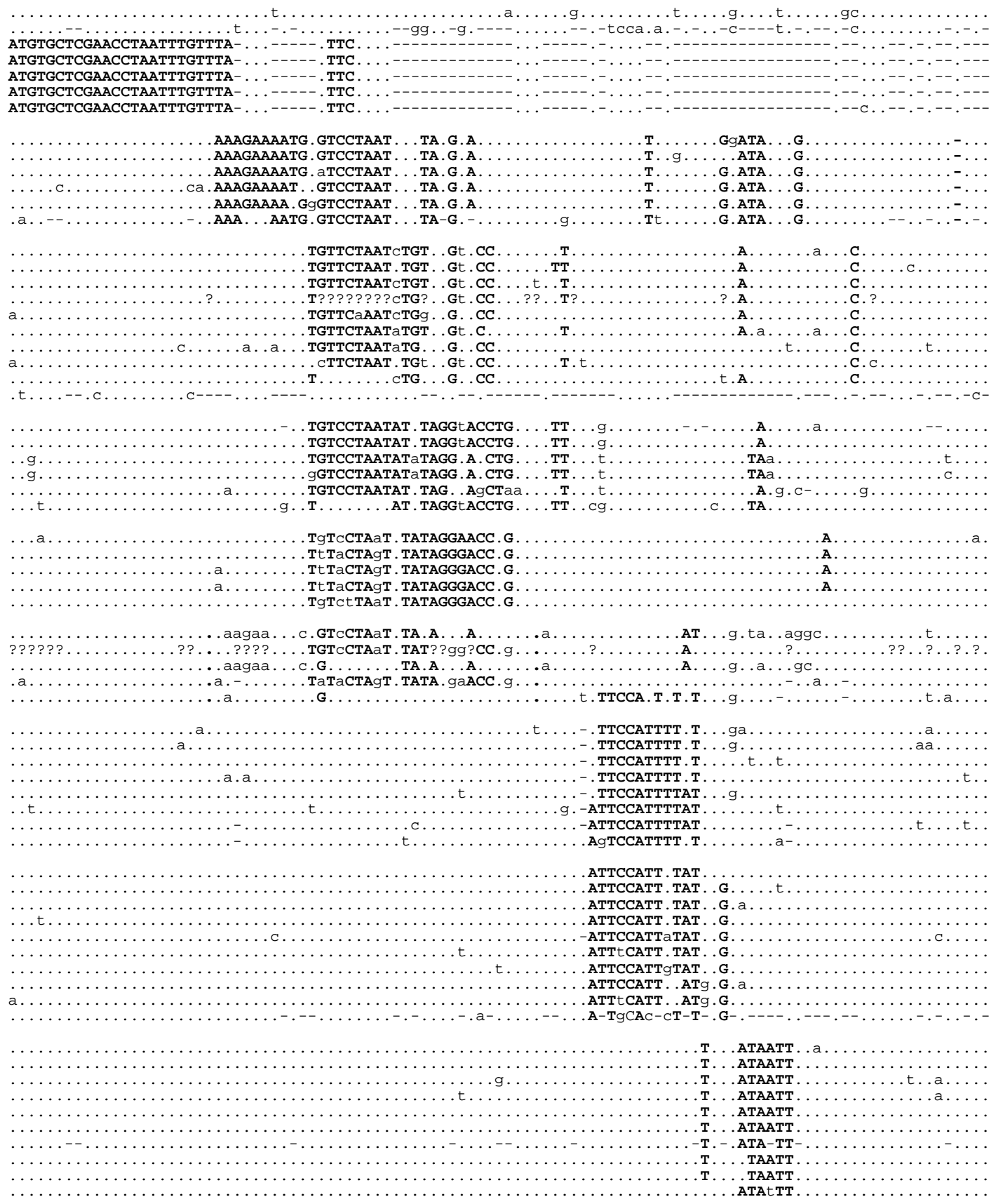




\section{FIGURE 4, p. 2}

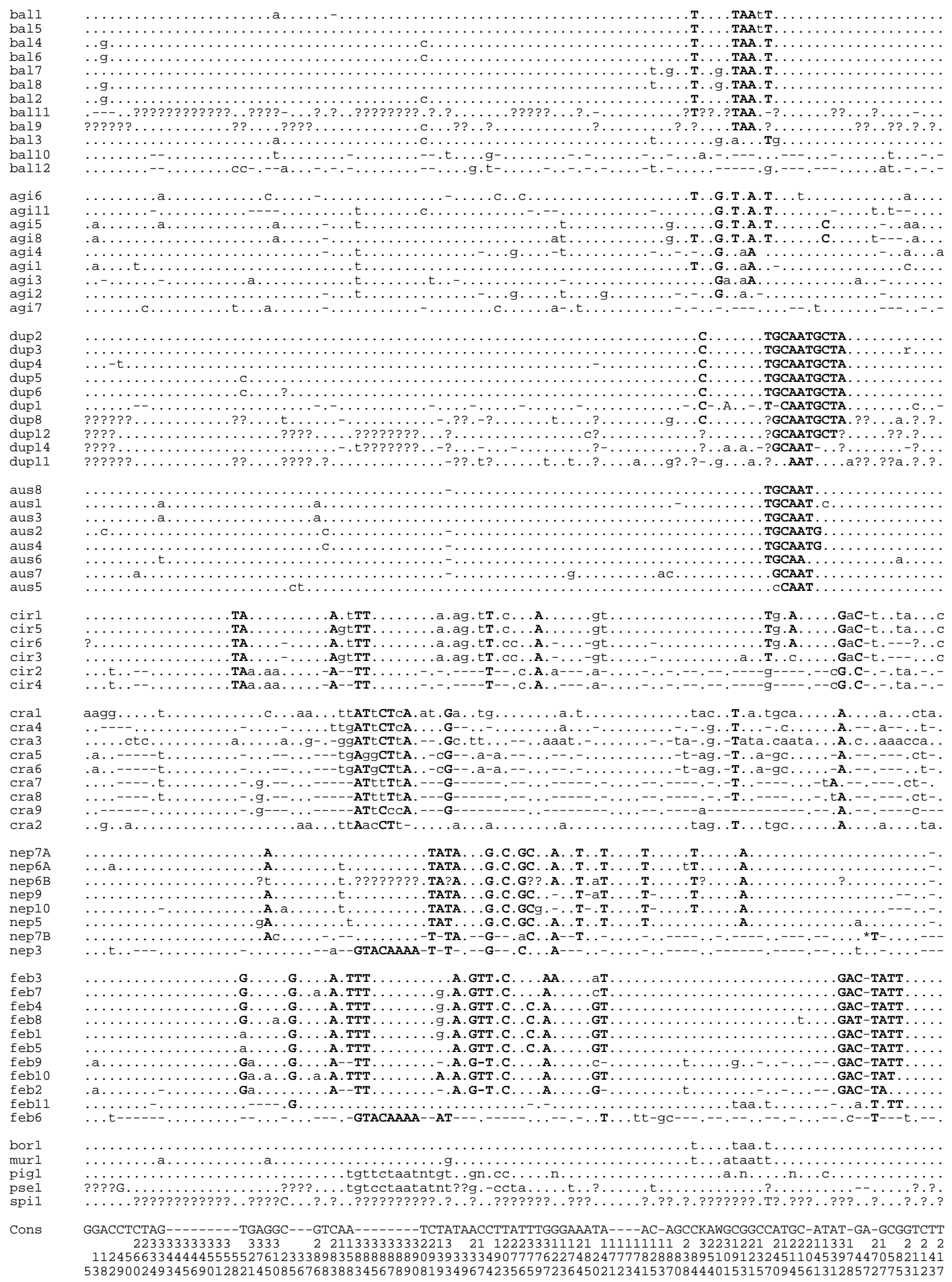




\section{FIGURE 5}

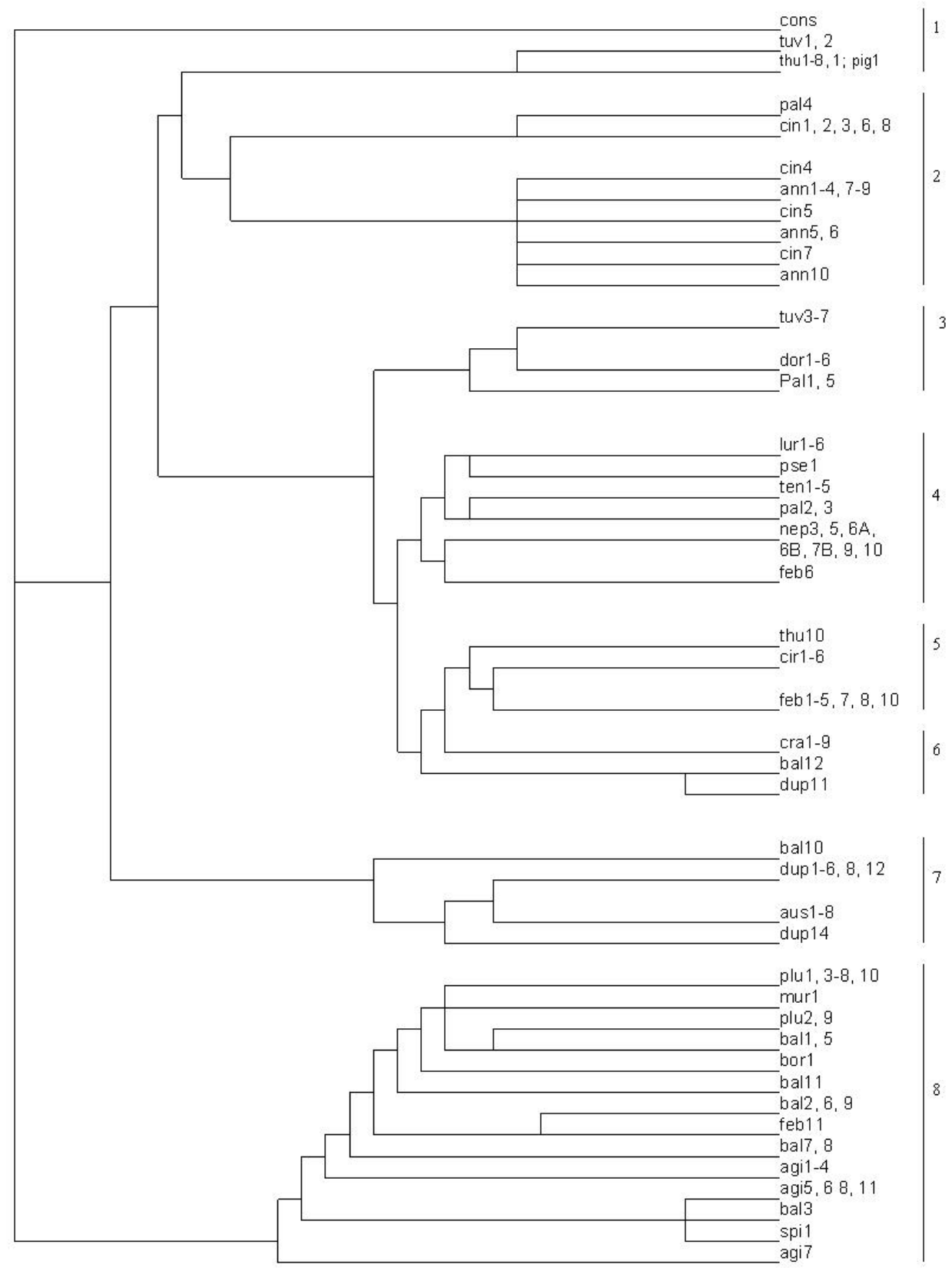




\section{FIGURE 6}

CTRT1 ACAATAGATTCTGTTCTAATTTGAATCGCAGTGCCTCCAGC NLRCth 1 AATCCGTAAATGAAATCAAAATTAAGAGACAAAAGGCAAAT

CTRT1 TGAAAGGCTGGGCCCTTCACACATTATATATATATATACT NLRCth 1 TGAATGCCTGAAACCTTAAAACAAAAATGCAACAAAATGT 